\title{
Effectiveness of School-Based Interventions in Reducing Unintentional Childhood Injuries: A Cluster Randomized Trial
}

\author{
Ramesh Holla $^{1}$, BB Darshan ${ }^{1}$, Bhaskaran Unnikrishnan ${ }^{1}$, Nithin Kumar ${ }^{1}$, Anju Sinha ${ }^{2}$, Rekha Thapar ${ }^{1}$, \\ P Prasanna Mithra $^{\mathbf{1}}$, Vaman Kulkarni ${ }^{1}$, Archana Ganapathy ${ }^{1}$, Himani Kotian ${ }^{1}$ \\ From Departments of ${ }^{1}$ Community Medicine, Kasturba Medical College, Mangalore (Manipal Academy of Higher Education), \\ Karnataka; and ${ }^{2}$ Division of Reproductive, Maternal and Child Health, Indian Council of Medical Research, Ansari Nagar, \\ New Delhi. \\ Correspondence to: Dr Ramesh Holla, Associate Professor, Department of Community Medicine, Kasturba Medical College, \\ Mangalore (Manipal Academy of Higher Education), Karnataka, India. ramesh.holla@manipal.edu \\ Received: November 09, 2020; Initial review: December 15, 2020; Accepted: February 04, 2021
}

Objective: To evaluate the effectiveness of school-based interventions in promoting child safety and reducing unintentional childhood injuries.

Methods: This cluster randomized trial with 1:1 allocation of clusters to intervention and control arm was conducted in the public and private schools of Dakshina Kannada district, Karnataka, over a period of 10 months. Study participants included children from standard 5-7 in schools selected for the study. 10 schools that could accommodate 1100 students each, were randomly allocated to the interventional and control arm. A comprehensive child safety and injury prevention module was developed based on the opinions of school teachers through focus group discussions. This module was periodically taught to the students of intervention arm by the teachers. The children in control arm did not receive any intervention. Outcome was

Trial registration: CTRI/2018/02/011765

$\mathrm{U}$ nintentional injuries specifically cause up to 950,000 deaths among children under 18 years annually [1] and more than half of these deaths are reported from Sub-Saharan Africa and South Asia [2]. Aside from mortality, accidental injuries can also lead to long-lasting emotional, physical, behavioral and developmental disabilities in children, which in turn could adversely affect the health and socio economic aspects of a nation [3].

Prevention of injuries has been classified into three strata of primary, secondary and tertiary prevention, as per a model suggested by World Health Organization [4]. The above-suggested WHO model can be incorporated while designing an effective school-based injuryprevention program. This can be used to address the policies and procedures, capacity building of school teachers, the physical environment of the school, and the curriculum in a coordinated manner.

There is little existing evidence to prove that educational interventions alone are sufficient in reducing assessed by determining the incidence of unintentional injuries and type of injuries from the questionnaire used at the baseline, and at the end of three, six, and ten months.

Results: Unintentional injuries declined progressively from baseline until the end of the study in both the interventional arm (from $52.9 \%$ to $2.5 \%$ ) and control arm (from $44.7 \%$ to $32 \%$ ) [AOR $(95 \% \mathrm{Cl}) 0.458(0.405-0.518) ; P$ value $<0.001]$. The decline in incidence of injuries in the interventional arm was higher than that in the control arm $(50.4 \%$ vs $12.7 \% ; P<0.001)$.

Conclusion: School based educational intervention using child safety and injury prevention modules is effective in reducing unintentional injuries among school children over a 10-month period.

Keywords : Education, Fall, Prevention, School health.

Published online: February 19, 2021; PII: S097475591600292

the incidence of unintentional injuries [5]. Further studies are required to evaluate the impact of school-based interventions on injury occurrence as current studies only show a weak association between the two [5]. Thus, this study was conducted to evaluate the effectiveness of school-based interventions in promoting child safety and reducing unintentional injuries.

\section{METHODS}

The study was conducted in the public and private schools of Dakshina Kannada district, Karnataka, over a period of 10 months from July, 2017 to March, 2018. It was a cluster randomized trial with 1:1 allocation of clusters into intervention arm and control arm, where schools are considered as clusters. After excluding schools based on their willingness to participate and existing participation in any child safety and injury prevention program, randomization of schools was done to accommodate 10 schools in the intervention arm and 10 schools in the control arm by simple random method. Due representation was provided to both public and private schools in both 
arms. The study participants included 1100 children from standard 5-7 in the schools selected for the study. We assumed there would be 40 students in each section of these standards. By enrolling all the students of a particular section, we would be enrolling 120 students from each cluster for the study. Selection of a section for a particular class was done by adopting simple random technique.

The sample size for the study was calculated by considering a prevalence of $23 \%$ childhood injuries as per a previous study [6]. The proposed intervention was considered effective if it reduced the incidence of injury to $15 \%$. Hence, to account for the $8 \%$ reduction as significant at $90 \%$ power, $5 \%$ level of significance and at two-sided test, the sample size was calculated to be 503 in each arm. As it was a cluster-randomized trial, we presumed a design effect of 2 and the sample size was 1006. As we anticipated a maximum of $10 \%$ loss during the follow-up period of 10 months; the final sample size was calculated to be 1107 in each arm.

A comprehensive child safety and injury prevention module was then developed based on the opinions of school teachers from both urban and rural settings through focus group discussions. Later, subject experts validated the contents of the module. This comprehensive pictorial module consisted of child safety and measures to be taken by the children for the prevention of unintentional childhood injuries due to road traffic accidents, fall, burns, drowning, poisoning, animal related and other domestic causes.

Two teachers (including one physical training/sports teacher) from each school of the interventional arm were trained using this module. The teachers then taught the children on a periodic and regular basis for the duration of the study, using an instruction manual for modular teaching (25-30 hours on an average was spent per school). The students in the control arm received the comprehensive modular training after the end of the final data collection. While imparting this modular training, emphasis was given for child safety and injury prevention strategies to be inculcated by the children.

The tool used for data collection was a semi-structured questionnaire developed based on World Health Organization guidelines for conducting community surveys on injuries and violence [7]. This captured the incidence of unintentional injuries and the type of injuries among schoolchildren of both arms in the preceding three months. The same questionnaire was administered for both the groups at baseline, and at three, six, and ten months of the study. Outcome was assessed by the same set of investigators at each point of time in both intervention and control arm students.
Clearance was obtained from the institutional ethics committee and permission was taken from the Block Education Office. Due clearance was also obtained from the school principals where the study was conducted. As the study participants were children younger than 18 years, a written informed consent was obtained from their parents before enrolment into the study. Assent from the students were also obtained. Confidentiality and anonymity was maintained throughout the study.

Statistical analysis: All the data collected in the field were managed at the central coordinating site. The variables were coded and entered into Statistical Package for Social Sciences Version 25.0 (IBM Corp). Descriptive statistics and inferential statistics ( $Z$ test for difference in two proportions, and generalized estimation equations (GEE) was used to test the overall effectiveness of the intervention across the groups with time) were used to express the results. $P<0.05$ was considered statistically significant.

\section{RESULTS}

Out of 2327 children who were enrolled into the study at baseline, 1177 children were in the interventional arm and 1150 were in the control arm (Fig. 1). The baseline data is provided in Table $\mathbf{I}$.

Incidence of unintentional childhood injuries among schoolchildren of interventional and control group during the study period is shown in Table II. Nearly half of the study participants of the intervention (52.9\%) and control (44.7\%) group had injuries in the preceding 3 months at the baseline. The incidence of injuries declined progressively from baseline until the end of the study among children in both the groups [Adjusted OR (95\% CI) 0.46 (0.40-0.52; $P<0.001]$ (Fig. 2).

The extent of decline in incidence of injuries from the start of the study till the end in the interventional arm was higher than in the control arm $(50.4 \%$ vs $12.7 \% ; P<0.001)$.

Table I Baseline Characteristics of Study Participants $(N=\mathbf{2 3 2 7})$

\begin{tabular}{llr}
\hline Characteristics & $\begin{array}{l}\text { Intervention group } \\
(n=1177)\end{array}$ & $\begin{array}{r}\text { Control group } \\
(n=1150)\end{array}$ \\
\hline $\begin{array}{l}\text { Male sex } \\
\text { Class }\end{array}$ & $658(55.9)$ & $500(43.5)$ \\
5 th & $367(31.2)$ & $362(31.5)$ \\
6 th & $306(26.0)$ & $501(43.5)$ \\
7 th & $504(42.8)$ & $287(25.0)$ \\
Urban locality & $656(55.7)$ & $314(27.3)$ \\
Government school & $507(43.1)$ & $408(35.5)$ \\
\hline Values in no.(\%). & &
\end{tabular}


Table II Incidence of Unintentional Childhood Injuries

\begin{tabular}{llr}
\hline Unintentional injury & Intervention group & Control group \\
\hline Baseline & $623(52.9)$ & $514(44.7)$ \\
3 mo & $224 / 1179(19.0)$ & $382 / 1123(34.0)$ \\
6 mo & $107 / 1184(9.0)$ & $442 / 1175(37.6)$ \\
End line & $29 / 1169(2.5)$ & $356 / 1113(32.0)$ \\
\hline
\end{tabular}

Incidence based on generalized estimating equations (GEEs). Values in $n / N(\%)$. Adjusted OR $(95 \% C I)=0.45(0.40-0.52), P<0.001$.

Various causes of unintentional childhood injuries across both groups throughout the duration of the study is depicted in Suppl. Table I. Fall was the most common cause of injury among children of interventional (56.8\%) and control group $(46.7 \%)$ at baseline. Decline in the incidence of unintentional injuries was observed in both the groups across all categories.

\section{DISCUSSION}

We found that the incidence of unintentional injuries among students in both the control arm and interventional arm decreased compared to baseline incidence. However, the extent of decrease was much greater in the interventional arm. While comparing incidences in both groups across specific categories, the number of children who

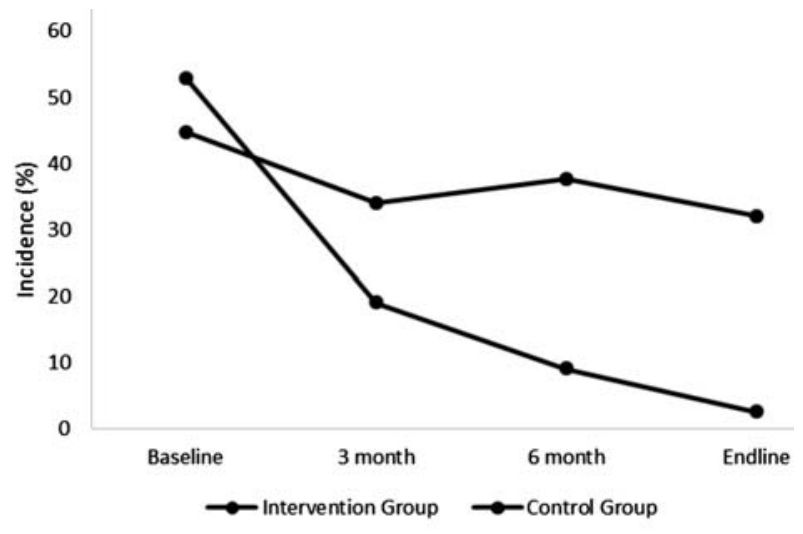

Fig. 2 Trends in incidence of unintentional childhood injuries over 10 months.

sustained injuries from road traffic accidents, falls and others decreased to a larger extent in the interventional group compared to the control group with the biggest reduction noted in falls.

A randomized pre-test and post-test comparative design study, 'Think First for Kids' [8] conducted among grade 1, 2 and 3 students, evaluated the outcome of an injury prevention program. The results of this study showed that students in the interventional group had lesser self-

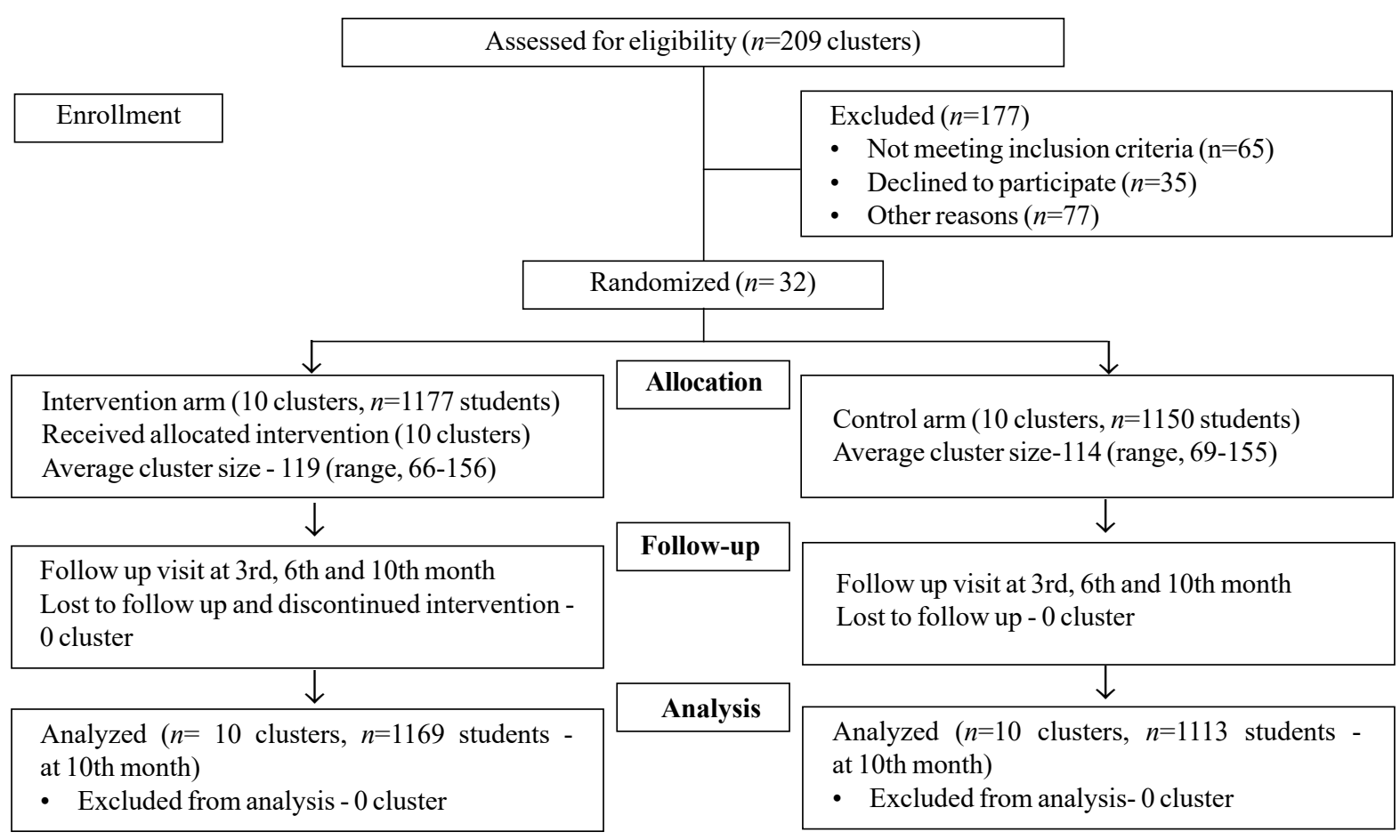

Fig. 1 Flow diagram of the study. 


\section{WHAT THIS STUDY ADDS?}

- A school-based educational intervention is effective in reducing the incidence of unintentional childhood injuries among school children.

reported high-risk behaviors, and increased knowledge about 'safe' behaviors to avoid injuries as compared to students in the control arm. In another study in rural China [9], a multi-level educational interventional model (open letter about security instruction distributed to parents, children's injury-avoidance poster put up at schools, and multimedia resource-aids for health education) improved knowledge and safety attitudes among students in the intervention arm as compared to the control arm.

It is interesting to note that the incidence of unintentional injuries decreased among children in the control group as well. We hypothesize that this could be due to a combination of various factors. This includes the learning curve of the child after experiencing an unintentional injury and knowledge gained over time from other sources such as parents or public health awareness campaigns.

From our study we also noted that the biggest reduction in unintentional injuries was in the category of falls among children in the interventional arm. The educational module imparted knowledge on safe behaviors at home and while playing outdoors. There were pictorial representations of scenarios which most-likely lead to falls such as playing on escalator and climbing trees. Another study by Morrongiello and Matheis [10] used a similar educational intervention and it was shown to reduce falls, particularly in the playground, through the 'practice what you preach' project. Children had less risktaking behavior and more safe practices after the intervention.

Unintentional injuries due to road traffic accidents also considerably reduced in the interventional group as compared to the control group. Pictorial representations of Dos and Don'ts related to Road safety was used to educate children every week. Another public school based educational intervention to improve attitudes, increase knowledge and change unsafe road practices was implemented in four schools in Mexico among 219 children and teenagers [11]. A significant improvement in the attitude, practices and knowledge of involved students were seen. The number of students suffering from burns decreased significantly in the interventional group while it remained constant in the control group, showing the effectiveness of the educational module in this area. A cluster randomized controlled trial evaluating an injury prevention program "Risk Watch' in 20 primary schools among 459 children aged 7-10 years in Nottingham, UK showed similar results [12]. At the end of this one-year injury prevention program, it was effective in increasing few aspects of children's knowledge of fire and burn prevention skills, although it had little effect on selfreported safety behaviors, unlike our study.

The main limitation of our study is that it is a single centric study and had a short duration of follow-up. The results obtained regarding the prevention of unintentional injuries among children using educational interventions cannot be extrapolated until further multi-centric studies show the same results. As this school based intervention using child safety and injury prevention module was found to be effective in reducing the incidence of unintentional injuries; this modular intervention can be considered for incorporating it in the school curriculum, after obtaining evidence from well-planned multi-centric studies incorporating a longer follow-up.

To conclude, the school based educational interventions using the child safety and injury prevention module have significantly reduced the incidence of unintentional injuries among children in the intervention arm when compared to students of control arm where such educational interventions were not given.

Acknowledgements: Mr. Laxminarayana Acharya and Ms. Mamatha, Medical Social Workers and Ms. Shika J, Data Entry Operator for successful completion of the project.

Note: Supplementary material related to this study is available with the online version at $w w w$.indianpediatrics.net

Ethics clearance: Institutional Ethics Committee of Kasturba Medical College, Mangalore; No. IEC KMC MLR 12-14/285, dated 17 December, 2014.

Contributors: RH, DB: Concept and design, analysis, interpretation of data, drafting the article; BUK: Concept and design, interpretation of data, drafting the article, revising it critically; $\mathrm{VK}$, NK: Interpretation of data, revising it critically for important intellectual content; RT, PM: Study design, interpretation of data, revising it critically for important intellectual content; AS: Revising the manuscript critically for important intellectual content and critical interpretation of the data captured; AG: Analysis of the data, drafting the manuscript and proof reading; HK: Data analysis and critical revision of the results. All authors approved the final version of manuscript, and are accountable for all aspects related to the study.

Funding: Indian Council of Medical Research;

Competing interests: None stated. 


\section{REFERENCES}

1. Orton E, Whitehead J, Mhizha-Murira J, et al. Schoolbased education programmes for the prevention of unintentional injuries in children and young people. Cochrane Database Syst Rev. 2016;12:CD010246.

2. Mahapatra T. Public health perspectives on childhood injuries around the world: A Commentary. Ann Trop Med Public Health 2015;8:233-4.

3. Sinha AP, Sarma S, Kamal R, Gupta P, Amritanshu. Prevention of unintentional childhood injuries in India: An Indian Council of Medical Research (ICMR) initiative. EC Paediatrics. 2020;9:143-48.

4. Barcelos RS, Del-Ponte B, Santos IS. Interventions to reduce accidents in childhood: a systematic review. J Pediatr (Rio J). 2018;94:351-67.

5. Centers for Disease Control and Prevention. School Health Guidelines to Prevent Unintentional Injuries and Violence. MMWR Recomm Rep. 2001;50:1-73.

6. Mahalakshmy T, Dongre AR, Kalaiselvan G. Epidemiology of childhood injuries in rural Puducherry, South India. Indian J Pediatr. 2011;78:821-5.

7. Seth D, editor. Guidelines for Conducting Community
Surveys on Injuries and Violence. World Health Organization. Available from: https://apps.who.int/iris/handle/ 10665/42975

8. Gresham LS, Zirkle DL, Tolchin S, Jones C, Maroufi A, Miranda J. Partnering for injury prevention: evaluation of a curriculum-based intervention program among elementary school children. J Pediatr Nurs. 2001;16:79-87.

9. Cao BL, Shi XQ, Qi YH, et al. Effect of a multi-level education intervention model on knowledge and attitudes of accidental injuries in rural children in Zunyi, Southwest China. Int J Environ Res Public Health. 2015;12: 3903-14.

10. Morrongiello BA, Mark L. Practice what you preach: Induced hypocrisy as an intervention strategy to reduce children's intentions to risk take on playgrounds. J Pediatr Psychol. 2008;33:1117-28.

11. Treviño-Siller S, Pacheco-Magaña LE, Bonilla-Fernández $\mathrm{P}$, et al. An educational intervention in road safety among children and teenagers in Mexico. Traffic Inj Prev. 2017;18:164-170.

12. Kendrick D, Groom L, Stewart J, et al. Risk watch: Cluster randomised controlled trial evaluating an injury prevention program. Inj Prev. 2007;13:93-8.

\section{CLIPPINGS}

\section{丹}

Efficacy of adjunctive zinc in improving the treatment outcomes in hospitalized children with pneumonia: $\mathbf{A}$ randomized controlled trial ( $J$ Trop Pediatr. 2020; $66: 419-27)$

Pneumonia is still one of the largest contributors to under-five mortality in developing country like ours. Zinc is used by health professionals as an adjunct treatment in children with pneumonia. This is a randomized, double blind placebo controlled trial conducted on hospitalized children with pneumonia with the aim to assess the efficacy of adjunctive zinc supplementation on the treatment outcomes of pneumonia. Ninety one children (2-6 months) were randomly received either zinc bis-glycinate $(15 \mathrm{mg}$ elemental zinc) or placebo, twice daily. The time to resolution of clinical pneumonia was significantly shorter in the zinc group than the placebo, and the hospitalization period as well as time to resolution of fever were significantly shorter in the zinc group. Due to small number of enrolled participants, the generalizability of the study on a global scale might be affected. Therefore, larger randomized trials are recommended for future investigations, as well as various variables that may influence the outcomes should be considered in future trials.
Skin manifestations of COVID-19 in children: Part 1, Part 2, Part 3. (Clin Exp Dermatol. 2021;46:444-72)

The current COVID-19 pandemic is caused by SARS-CoV-2. The initial recognized symptoms were respiratory, sometimes culminating in severe respiratory distress requiring ventilation, as time has passed, other symptoms have also been recognized. The initial reports of cutaneous manifestations were from Italian dermatologists, probably because Italy was the first European country to be heavily affected by the pandemic. The overall clinical presentation, course and outcome of SARS-CoV-2 infection in children differ from those in adults as do the cutaneous manifestations of childhood. In this extensive review, divided into three parts, the current knowledge on the cutaneous manifestations of COVID-19 in children are summarized after thorough and critical review of articles published in the literature and from the personal experience of a large panel of paediatric dermatologists in Europe. In Part 1, first and most widespread cutaneous manifestation of COVID-19, chilblain-like lesions are discussed. In Part 2, other cutaneous manifestations are reviewed, including erythema multiforme, urticaria and Kawasaki diseaselike inflammatory multisystemic syndrome; while in Part 3, the histological findings of COVID-19 manifestations are discussed, and the testing and management of infected children, for both COVID-19 and any other pre-existing conditions.

SUMAIRA KHALIL sumairakhalil@yahoo.com 